steps and beds ranked among the most frequent safety hazards. Injuries were most frequently triggered by beds as well as by flooring covering or floor surfaces (rugs, mats).

Conclusions Effective accident prevention requires both an individual risk assessment and individual counselling in the home setting.

\title{
0808 PRODUCT-RELATED INJURIES IN ELDERLY PERSONS
}

G Ellsaesser*, A Krampe Correspondence: Brandenburg Regional Public Health Office, Whsdorfer Platz 3, Zossen, D-15806, Germany

10.1136/ip.2010.029215.808

Background In order to improve the statistical basis for estimating the number of injuries across all age groups, the Public Health Institute of Brandenburg has been developing a hospital related injury recording system according to the European Injury Data Base.

Method In a catchment area of about 150000 inhabitants (Land Brandenburg) all injured patients admitted to hospital and all outpatients are recorded on 1 day of the week (24 h) according to the European IDB standard. The 12 month prevalence was counted using the official German Hospital Diagnosis Statistics. The share of inpatient sample cases in all hospital patients living in the catchment area according yields as grossing-up factor for the number of outpatient sample cases per year.

Results Within a 2-year period 2007-2008 a total of 2149 injured patients at the age of 65 years and older received inpatient or outpatient treatment. The injury rate rose sharply in persons at the age of 80 years and older (47.5 per 1000). In this age group almost one in two falls was caused by the affected person itself. Indoor areas and particularly flooring, stairs, 